\title{
Von zentralen Orten und inneren Städten
}

\author{
Gregor Prinzensing
}

Online publiziert: 31. Juli 2015

(C) Springer-Verlag Berlin Heidelberg 2015

Liebe Leserinnen und Leser,

mit der neuesten Ausgabe der „Raumforschung und Raumordnung“ präsentieren wir Ihnen wieder einige lesenswerte Facetten aus aktuellen raumwissenschaftlichen Forschungen.

Im ersten Beitrag „Die innere Stadt als Wohnstandort der jungen Alten?“ widmen sich Anne Rabe und Uta Hohn am Beispiel von Wohnstandortentscheidungen von ,jungen Alten“ der Frage, inwiefern die sogenannte „Innere Stadt“ inzwischen ein zunehmend attraktiver Wohnstandort für diese Bevölkerungsgruppe ist. Die ,innere Stadt“ schließt nach einer Definition des Bundesamts für Bauwesen und Raumordnung (BBR) neben der eigentlichen Innenstadt (der klassisch verstandenen City) auch diejenigen Stadtbezirke am Innenstadtrand mit ein, die unmittelbar an die Kernstadt angrenzen. Die Autorinnen untersuchen ihre Fragestellung empirisch anhand von Zuzügen in die Stadt Bonn im Zeitraum zwischen 2010 und 2012. Das Ergebnis ihrer Studie ordnet sich auf den ersten Blick in kurrente Reurbanisierungserscheinungen ein. Neben einer zunehmenden Anziehungskraft innerstädtischen Wohnens für die ,jungen Alten" sind jedoch auch kleinräumliche Unterschiede zu verzeichnen, die für die Attraktivität der Inneren Stadt als Wohnstandort entscheidend sind. Insgesamt wird von den ,jungen Alten“ anscheinend vor allem das Wohnen am Innenstadtrand präferiert; die Innenstadt scheint demgegen-

G. Prinzensing $(\square)$

Akademie für Raumforschung und Landesplanung (ARL), Leibniz-Forum für Raumwissenschaften, Hohenzollernstraße 11, 30161 Hannover, Deutschland

E-Mail: prinzensing@arl-net.de über in erster Linie attraktiv als Ort zum Einkaufen und für die Freizeitgestaltung. Aber auch innerhalb des Innenstadtrands muss den Befunden zufolge noch einmal entlang von Lebensstil- und Einkommensunterschieden zwischen attraktiven und weniger attraktiven Lagen unterschieden werden. Die Untersuchung arbeitet so nicht nur einer differenzierteren Betrachtung der Gesamtstadt bei der Untersuchung von Reurbanisierungsphänomenen zu, sondern leistet auch einen Beitrag zur Strukturierung und Aufgliederung in Bezug auf das Konzept der „Inneren Stadt“.

Hendrik Kohl und Simone Strambach erforschen in ihrem Beitrag Zusammenhänge von „Mobilitätsdynamiken und Wissensarbeit“ und diskutieren den „Wandel berufsbedingter zirkulärer Mobilität". Zwar ist die wissensbasierte Ökonomie, zwar ist auch das Konzept wissensintensiver (immaterieller) Tätigkeiten inzwischen eine feste Größe in den Debatten um die Richtung des neueren sozioökonomischen Strukturwandels. Allerdings stehen Untersuchungen über die Auswirkungen dieser Dynamik auf das Mobilitätsverhalten von Wissensarbeitenden offenbar erst am Anfang. Zumindest auf den ersten Blick erscheint die Annahme plausibel, dass kreative bzw. immateriell-geistige Tätigkeiten, da sie nicht an feste Objekte gebunden sind, sich auch gut auf Medien der modernen IuK-Technologien stützen lassen und deshalb im Prinzip räumlich entgrenzt durchgeführt werden können. Dies würde es zulassen, im Bereich der Wissensarbeit von tendenziell eher sinkenden Mobilitätsanforderungen auszugehen. Die Mobilitätsforschung der vergangenen Jahre verzeichnet dagegen bis in die Gegenwart hinein ein kaum gebremstes Anwachsen von Mobilität. Hier versuchen die Autoren, durch die Zusammenführung von Erkenntnissen aus der interdisziplinären Mobilitätsforschung und der raumbezogenen Innovations- und Wissensforschung die unterschiedlichen räumlichen und zeitlichen Bindungen von Wissensarbeit genauer zu ermitteln und 
die komplexen Implikationen für die berufsbedingte zirkuläre Mobilität von Wissensarbeitenden zu analysieren. Sie kommen zu dem Schluss, dass der empirisch sichtbare quantitative Zuwachs berufsbedingter Mobilität auch eine qualitative Seite hat. Wissensintensive Tätigkeiten sind offenbar mit sich dynamisch und fortlaufend ändernden (fluiden) Anforderungen an die berufsbedingte zirkuläre Mobilität in verschiedenen raum-zeitlichen Dimensionen verbunden.

Im Anschluss daran untersuchen Uwe Neumann, Thomas K. Bauer, Rüdiger Budde und Martin Micheli in ihrem Beitrag „Immobilienmarkteffekte des Emscherumbaus?“ die Immobilienpreiseffekte von großräumigen Infrastrukturmaßnahmen in regionalen Kontexten. Untersuchungsgebiet ist das „Neue Emschertal“, als Vergleichsregionen dienen benachbarte Gemeinden des Emscherumbau-Gebiets (sonstige Emscherregion, Ruhrtal). Auf der Basis sogenannter hedonischer Preisfunktionen wird mittels georeferenzierter Immobilienhandelsdaten kleinräumig analysiert, wie sich die mit dem Emscherumbau verbundene Aufwertung örtlicher Standortqualitäten auf die lokalen Immobilienpreise im Zeitraum 2007 bis 2011 auswirkte. Bei der Schätzung hedonischer Preisfunktionen wird der Einfluss von Qualitätsunterschieden auf den Preis von Gütern festgestellt, indem Preise für Güter mit bestimmten Eigenschaften verglichen werden mit Preisen für Güter mit davon abweichenden Eigenschaften. Auf diese Weise können regressionsanalytisch jene Preisunterschiede ermittelt werden, die sich allein auf Qualitätsunterschiede zurückführen lassen. Die gewonnenen empirischen Resultate legen für das Untersuchungsgebiet den Schluss nahe, dass der Emscherumbau in den davon berührten Nachbarschaften bisher keine statistisch signifikanten Mietpreiseffekte zur Folge hatte, während die Umbaumaßnahmen bei Eigentumswohnungen zum
Werterhalt beigetragen haben und somit in einem gewissen Umfang positive Preiseffekte erkennbar sind.

Im vierten Beitrag schließlich legen Stefan Greiving, Florian Flex und Thomas Terfrüchte eine „Vergleichende Untersuchung der Zentrale-Orte-Konzepte in den Ländern und Empfehlungen zu ihrer Weiterentwicklung" vor. Die Landes- und Regionalplanungen aller Flächenländer Deutschlands enthalten zentralörtliche Konzeptionen als integralen Bestandteil. Vor dem Hintergrund, dass in vielen Teilen Deutschlands ein gleichsam demographisch induzierter Problemdruck besteht, war eine aktuelle und umfassende Übersicht zum Status quo in den Bundesländern ein Desiderat. Der Beitrag bietet hier einen strukturierten Überblick und weist auf Probleme in der Anwendung hin. Im Ergebnis einer vergleichenden Bestandsaufnahme zeigt sich, dass es das eine Zentrale-Orte-Konzept nicht (mehr) gibt. In den Ländern haben sich mittlerweile unterschiedliche Auffassungen hinsichtlich der elementaren Bestandteile und Begrifflichkeiten sowie auch unterschiedliche Ansätze beim Steuerungshandeln herausgebildet. Für die Weiterentwicklungen der Zentrale-Orte-Konzeptionen kommt es nach Ansicht der Autoren zunächst auf eine empirische Überprüfung des jeweils vorfindbaren Zentrale-Orte-Systems an. Dafür sei ein politisch legitimiertes Zielsystem erforderlich, aus dem sich die Kriterien für die empirische Überprüfung und Einstufung von Zentralen Orten ableiten lassen. Vor diesem Hintergrund seien sodann auch raumstrukturell differenzierte Einstufungen begründbar, die ausstattungsgleiche Orte im Kontext unterschiedlicher Raumstrukturen ungleich behandeln.

Wir wünschen Ihnen wie immer eine aufschlussreiche Lektüre. 